\title{
Parenting, community, and religious predictors of positive and negative developmental outcomes among Muslim adolescents
}

\begin{abstract}
Despite existing research on the contribution of social context and religiosity to adolescent behavioral outcomes, few studies have attempted to explore this topic among Muslim adolescents in non-Western settings, looking at both positive and negative outcomes. In response to this gap, the current study explored the effects of three dimensions of developmental assets (positive parenting, community support, and religiosity) on risk, prosocial, and thriving behaviors among Muslim adolescents $(\mathrm{N}=895)$ from Malaysia. Hierarchical regression results revealed positive parenting as the greatest protective factor against risk behavior, religiosity as the most significant promotive factor of prosocial behaviors, and community support as the greatest contributor to adolescent thriving. In the final model, unique effects varied by outcome. The findings support the importance and universality of multiple evels of developmental assets for youth development, and highlight the need to better understand their interaction in non-Western cultural contexts.
\end{abstract}

Keyword: Developmental outcomes; Positive youth development; Muslim youth; Malaysia 